Proceedings of the

International Geometry Center

Vol. 9, no. 3-4 (2016) pp. 37-49

\author{
Vol. 9, no. 3-4 (2016) pp. $37-49$
}

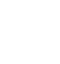

\title{
Топологічні властивості частково метричних просторів
}

\author{
Вадим Мироник, Володимир Михайлюк
}

\begin{abstract}
We study topological properties of partial metrics and partial metric spaces. In particular, we investigate relations between the regularity of a partial metric space and continuity type properties of the corresponding partial metric. For mappings with values in a partial metric space we obtain an analogue of a theorem on $G_{\delta}$-type of a set of the continuity points of mapping with values in a metrizable space and an analogue of a theorem on $F_{\sigma}$-measurability of a semicontinuous function.
\end{abstract}

\begin{abstract}
Анотація. Ми вивчаємо топологічні властивості часткових метрик і частково метричних просторів, зокрема, досліджуємо зв'язок між регулярністю частково метричних просторів і різними аспектами неперервності часткової метрики. Для відображень зі значеннями у частково метричних просторах ми одержуємо аналоги теореми про $G_{\delta}$-тип множини точок неперервності метризовнозначних відображень і теореми про $F_{\sigma}$-вимірність напівнеперервної функції.
\end{abstract}

\section{1. ВСТУП}

Поняття часткової метрики і частково метричного простору було введене С. Метьюсом [6], [7] у 1992 році. Це поняття виникло як певне послаблення поняття метричного простору і застосовувалось в дослідженнях семантики мов програмування, де виникають негаусдорфові топологічні моделі (дивись [10]).

Разом $з$ тим частково метричні простори дістали широке застосування в теорії нерухомої точки (Fixed Point Theory) і інтенсивно використовуються для різних узагальнень теореми Банаха про нерухому точку [9], [2], [3], [5], [1].

2010 Mathematics Subject Classification: Mathematics Subject Classification: 54D10, $54 \mathrm{E} 35$

Ключові слова: часткова метрика, частково метричний простір, напівнеперервність, регулярність, метризовність 
Слід зазначити, що загальні топологічні властивості частково метричних просторів у вищезгаданих роботах досліджені досить поверхово. Крім того, природно виникає питання про те, які з результатів з теорії метричних просторів переносяться без змін на випадок частково метричних просторів або мають свої аналоги у цьому загальнішому класі просторів.

В даній статті ми вивчаємо топологічні властивості часткових метрик, частково метричних просторів і зв'язок між ними. Також ми досліджуємо зв'язок між регулярністю частково метричних просторів і різними аспектами неперервності часткової метрики. Крім того, для відображень зі значеннями у частково метричних просторах ми доводимо аналоги теореми про $G_{\delta}$-тип множини точок неперервності метризовно-значних відображень і теореми про $F_{\sigma}$-вимірність напівнеперервної функції.

\section{2. ПРОСТшШ ТОПОЛОГІЧНІ ВЛАСТИВОСТІ І ПРИКЛАДИ ЧАСТКОВО МЕТРИЧНИХ ПРОСТОРІВ}

Означення 2.1. Функція $p: X^{2} \rightarrow \mathbb{R}$ називається частковою метрикою на множині $X$, якщо для довільних $x, y, z \in X$ виконуються наступні умови:

$\left(p_{1}\right) x=y \Leftrightarrow p(x, x)=p(x, y)=p(y, y) ;$

$\left(p_{2}\right) p(x, x) \leq p(x, y)$;

$\left(p_{3}\right) p(x, y)=p(y, x)$;

$\left(p_{4}\right) p(x, z) \leq p(x, y)+p(y, z)-p(y, y)$.

Нехай $(X, p)$ - частково метричний простір. Для кожного $x \in X \mathrm{i}$ $\varepsilon>0$ покладемо

$$
B_{p}(x, \varepsilon)=\{y \in X: p(x, y)<p(x, x)+\varepsilon\} .
$$

Система $\left\{B_{p}(x, \varepsilon): \varepsilon>0\right\}$ утворює базу околів деякої топології на просторі $X$, яка називається топологією частково метричного простору. При цьому всі множини $B_{p}(x, \varepsilon)$ є відкритими в частково метричному просторі $(X, p)$.

Легко бачити, що часткова метрика $p \in$ метрикою, якщо $p(x, x)=0$ для кожного $x \in X$. Крім того, у первісному означенні часткової метрики $з$ [7] вимагається невід'ємність функції $p$, тобто $p(x, y) \geq 0$ для всіх $x, y \in X$. Але при введенні топології, породженої частковою метрикою $p$, невід'ємність функції $p$ не використовується. Отже, з точки зору топології частково метричного простору $(X, p)$ невід'ємність функції $p$ є зайвою.

Будемо говорити, що точка $x$ в топологічному просторі $X$ відокремлюеться від точки $y \in X$, якщо існує окіл $U$ точки $x$ такий, що $y \notin U$. 
Твердження 2.2. Нехай $(X, p)$ - частково метричний простір. Тодi

(1) точка $x \in X$ відокремлюеться від точки $y \in X$ тодi $i$ тільки тодi, коли $p(x, y)>p(x, x)$;

(2) $(X, p)-T_{0}$-nростір;

(3) $(X, p)-T_{1}$-простір тодi $i$ тільки тодi, коли $p(x, y)>p(x, x)$ для довільних різних $x, y \in X$.

Доведення. (1) 3 означення топологічної структури простору $(X, p)$ випливає, що точка $x \in X$ відокремлюється від точки $y \in X$ тоді і тільки тоді, коли $p(x, y) \geq p(x, x)+\varepsilon$ для деякого $\varepsilon>0$, тобто коли $p(x, y)>p(x, x)$.

(2) Виберемо довільні різні $x, y \in X$. Тоді з умов $\left(p_{1}\right)$ і $\left(p_{2}\right)$ випливає, що $p(y, x)>p(x, x)$ або $p(x, y)>p(y, y)$. Залишилось використати (1).

Умова (3) випливає безпосередньо 3 (1).

Твердження 2.3. Нехай $(X, p)$ - частково метричний простір. Тодi функиія $p:(X, p)^{2} \rightarrow \mathbb{R}$ - сукупно напівнеперервна зверху на $X^{2} i$ неперервна відносно кожної змінної у всіх точках діагоналі

$$
\Delta=\{(x, x): x \in X\} .
$$

Доведення. Покажемо спочатку, що $p$ - сукупно напівнеперервна функція. Зафіксуємо $x_{0}, y_{0} \in X$ i $\varepsilon>0$. Використовуючи умову $\left(p_{4}\right)$ для довільних $x \in B_{p}\left(x_{0}, \varepsilon\right)$ і $y \in B_{p}\left(y_{0}, \varepsilon\right)$ одержимо

$$
\begin{aligned}
p(x, y) & \leq p\left(x, x_{0}\right)+p\left(x_{0}, y\right)-p\left(x_{0}, x_{0}\right) \\
& \leq p\left(x_{0}, x_{0}\right)+\varepsilon-p\left(x_{0}, x_{0}\right)+p\left(x_{0}, y_{0}\right)+p\left(y_{0}, y\right)-p\left(y_{0}, y_{0}\right) \\
& <p\left(x_{0}, y_{0}\right)+2 \varepsilon .
\end{aligned}
$$

Нарізна неперервність функції $p$ у точках діагоналі $\Delta$ випливає з нерівності

$$
0 \leq p(x, y)-p(x, x)<\varepsilon
$$

для довільних $x \in X, \varepsilon>0$ і $y \in B_{p}(x, \varepsilon)$.

Нехай $S$ - довільна множина. Через $l_{1}(S)$ ми позначаємо множину всіх функцій $x: S \rightarrow \mathbb{R}$ таких, що носій $\operatorname{supp} x=\{s \in S: x(s) \neq 0\}$ не більш, ніж зліченний і ряд $\sum_{s \in S} x(s)=\sum_{s \in \operatorname{supp} x} x(s)$ абсолютно збіжний.

Приклад 2.4. Наступні функції $p$ є частковими метриками на відповідних множинах $X$ :

(a) $X \subseteq \mathbb{R}$ i $p(x, y)=\max \{x, y\}$ для довільних $x, y \in X$;

(b) $X \subseteq \mathbb{R}$ i $p(x, y)=-\min \{x, y\}$ для довільних $x, y \in X$; 
(c) $X \subseteq L_{1}([0,1])$ i $p(x, y)=\int_{0}^{1} \max \{x(t), y(t)\} d \mu(t)$ для довільних $x, y \in X$

(d) $X \subseteq l_{1}(S)$ і $p(x, y)=\sum_{s \in S} \max \{x(s), y(s)\}$ для довільних $x, y \in X$.

Зауваження 2.5. Для довільного топологічного простору $X$ функція $f: X \rightarrow \mathbb{R}$ напівнеперервна зверху чи знизу, якщо $f$ неперервна, як функція зі значеннями у частково метричному просторі $(\mathbb{R}, p)$ з прикладу 2.4(a) чи (b) відповідно.

\section{3. МЕТРИКА, ПОРОДЖЕНА ЧАСТКОВОЮ МЕТРИКОЮ, І РЕГУЛЯРНІ ЧАСТКОВО МЕТРИЧНІ ПРОСТОРИ}

Нехай $(X, p)$ - частково метричний простір. Легко бачити [7], що функція $d_{p}: X^{2} \rightarrow \mathbb{R}$,

$$
d_{p}(x, y)=2 p(x, y)-p(x, x)-p(y, y)
$$

$\epsilon$ метрикою на $X$, яку ми називатимемо метрикою, породженою частковою метрикою $p$. Тотожне відображення $\pi_{p}:(X, p) \rightarrow\left(X, d_{p}\right)$, $\pi_{p}(x)=x$, ми називатимемо відображенням, асоиійованим з частковою метрикою $p$. Крім того, для кожного $x \in X$ i $\varepsilon>0$ покладемо

$$
B_{d_{p}}(x, \varepsilon)=\left\{y \in X: d_{p}(x, y)<\varepsilon\right\} .
$$

Зауважимо, що $B_{d_{p}}(x, \varepsilon) \subseteq B_{p}(x, \varepsilon)$ для всіх $x \in X$ i $\varepsilon>0$. Тому має місце наступний факт.

Твердження 3.1. Відображення $\pi_{p}^{-1}:\left(X, d_{p}\right) \rightarrow(X, p)$ неперервне.

Наступне твердження дає зв'язок неперервності асоційованого відображення $\pi_{p}$ з неперервністю часткової метрики.

Твердження 3.2. Для частково метричного простору $(X, p)$ i точки $x_{0} \in X$ наступні умови є рівносильними:

(1) часткова метрика р е неперервною в точиі $\left(x_{0}, x_{0}\right)$;

(2) звуження часткової метрики $p$ на діагональ $\Delta=\{(x, x): x \in X\}$ $\epsilon$ неперервним в точиі $\left(x_{0}, x_{0}\right)$;

(3) асочійоване відображсення $\pi_{p}:(X, p) \rightarrow\left(X, d_{p}\right)$ є неперервним в mочui $x_{0}$.

Доведення. Імплікація $(1) \Rightarrow(2)$ є очевидною.

$(2) \Rightarrow(3)$. Зафіксуємо $\varepsilon>0$ і знайдемо окіл $U_{1}$ точки $x_{0}$ в $(X, p)$ такий, що

$$
\left|p(x, x)-p\left(x_{0}, x_{0}\right)\right|<\frac{\varepsilon}{3}
$$


для кожного $x \in U_{1}$. Тепер для кожного $x \in U_{1} \cap B_{p}\left(x_{0}, \frac{\varepsilon}{3}\right)$ маємо

$$
d_{p}\left(x, x_{0}\right)=2\left(p\left(x, x_{0}\right)-p\left(x_{0}, x_{0}\right)\right)+\left(p\left(x_{0}, x_{0}\right)-p(x, x)\right)<2 \cdot \frac{\varepsilon}{3}+\frac{\varepsilon}{3}=\varepsilon .
$$

Отже, відображення $\pi_{p}$ неперервне в точці $x_{0}$.

$(3) \Rightarrow(1)$. Згідно з твердженням 2.3 достатньо довести, що функція $p$ напівнеперервна знизу в точці $\left(x_{0}, x_{0}\right)$. Зафіксуємо $\varepsilon>0$ і виберемо окіл $U$ точки $x_{0}$ в $(X, p)$ такий, що $d_{p}\left(x, x_{0}\right)<\varepsilon$ для кожного $x \in U$. Тоді використовуючи умову $\left(p_{2}\right)$ для довільних $x, y \in U$ одержимо

$$
\begin{aligned}
p(x, y) \geq p(x, x) & =\left(p(x, x)-p\left(x, x_{0}\right)\right)+\left(p\left(x, x_{0}\right)-p\left(x_{0}, x_{0}\right)\right)+p\left(x_{0}, x_{0}\right) \\
& \geq\left(p(x, x)-p\left(x, x_{0}\right)\right)+\left(p\left(x_{0}, x_{0}\right)-p\left(x, x_{0}\right)\right)+p\left(x_{0}, x_{0}\right) \\
& =-d_{p}\left(x, x_{0}\right)+p\left(x_{0}, x_{0}\right)>p\left(x_{0}, x_{0}\right)-\varepsilon .
\end{aligned}
$$

Твердження доведено.

Наслідок 3.3. Нехай $(X, p)$ - частково метричний простір. Тоді наступні умови рівносильні:

(1) часткова метрика р є неперервною;

(2) звуження часткової метрики $p$ на діагональ $\Delta=\{(x, x): x \in X\}$ $\epsilon$ неперервним;

(3) асоційоване відображення $\pi_{p}:(X, p) \rightarrow\left(X, d_{p}\right)$ є неперервним;

(4) топологічні простори $(X, p) i\left(X, d_{p}\right)$ збігаютъся.

Для топологічного простору $X$, функції $f: X \rightarrow \mathbb{R}$ і точки $x_{0} \in X$ через $\omega_{f}\left(x_{0}\right)$ ми позначаємо коливання функції $f$ в точці $x_{0}$. Тобто

$$
\omega_{f}\left(x_{0}\right)=\inf _{U \in \mathcal{U}} \sup _{u, v \in U}|f(u)-f(v)|,
$$

де $\mathcal{U}$ - система всіх околів точки $x_{0}$.

Наступна властивість для напівнеперервних функцій дійсної змінної, як функцій першого класу Бера, є добре відомою [8, Глава XV, § 3-4].

Твердження 3.4. Нехай $X$ - топологічний простір, $f: X \rightarrow[0,1]$ напівнеперервна зверху функиія $i \varepsilon>0$. Тоді існує ніде не щільна множина $A \subseteq X$ така, що $\omega_{f}(x)<\varepsilon$ для кожного $x \in X \backslash A$.

Доведення. Виберемо $n \in \mathbb{N}$ таким чином, щоб $\frac{1}{n}<\frac{\varepsilon}{4}=\delta$ і для кожного $k \in\{1, \ldots, n\}$ покладемо

$$
A_{k}=\left\{x \in X: \omega_{f}(x) \geq \varepsilon\right\} \cap f^{-1}\left(\left[\frac{k-1}{n}, \frac{k}{n}\right]\right) .
$$

Достатньо показати, що всі множини $A_{k}$ ніде не щільні.

Припустимо, що деяка множина $A_{k}$ щільна у непорожній відкритій множині $U \subseteq X$. Зафіксуємо $x_{0} \in U \cap A_{k}$ і виберемо відкритий окіл $U_{1}$ точки $x_{0}$ такий, що $f(x)<f\left(x_{0}\right)+\delta$ для кожного $x \in U_{1}$. Оскільки 
$\omega_{f}\left(x_{0}\right) \geq 4 \delta$, то існуе $x_{1} \in U \cap U_{1}$ таке, що $f\left(x_{1}\right)<f\left(x_{0}\right)-2 \delta \leq \frac{k-1}{n}-\delta$. Тоді для довільного околу $U_{2}$ точки $x_{1}$ існує $x_{2} \in U_{2} \cap U \mathrm{i}$, зокрема,

$$
f\left(x_{2}\right) \geq \frac{k-1}{n} \geq f\left(x_{1}\right)+\delta
$$

що суперечить напівнеперервності зверху $f$ у точці $x_{1}$.

Тепер з твердження 2.3, наслідку 3.3 і твердження 3.4 випливає наступний факт.

Наслідок 3.5. Нехай $(X, p)$ - частково метричний простір. Тодi icнуе множина $A$ першої категорії в $(X, p)$ така, що простори $(X \backslash A, p)$ $i\left(X \backslash A, d_{p}\right)$ збігаються, зокрема, $X \backslash A$-метризовний підпростір npocmopy $X$.

\section{4. РЕГУЛЯРНІСТЬ ЧАСТКОВо МЕТРИЧНИХ ПРОСТОРіВ}

Топологічний простір $X$ називатимемо регулярним в точці $x_{0} \in X$, якщо для довільного околу $U$ точки $x_{0}$ в $X$ існує замкнений окіл $V$ точки $x_{0}$ такий, що $V \subseteq U$. Як випливає з наслідку 3.3 , якщо відображення $\pi_{p}$ неперервне, то простір $(X, p)$ - метризовний, зокрема регулярний. Тому природно виникає питання про точковий варіант цієї властивості. Тобто чи випливає 3 неперервності в точці $x_{0}$ асоційованого відображення $\pi_{p}$ регулярність частково метричного простору $(X, p)$ в точці $x_{0}$ ? Разом 3 тим, постає питання про зворотній зв'язок. A саме, чи випливає з регулярності (в точці) простору $(X, p)$ неперервність (в точці) відображення $\pi_{p}$ ? Наступні два приклади показують, що ці питання мають негативні відповіді.

Твердження 4.1. Існуе частково метричний простір $(X, p)$ такий, що асоційоване відображення $\pi_{p}$ неперервне в точиі $x_{0} \in X$, але $(X, p)$ не є регулярним в точщі $x_{0}$.

Доведення. Нехай $S=\{0\} \cup \mathbb{N} \cup \mathbb{N}^{2}$. Побудуємо сім'ю $\left(x_{s}: s \in S\right)$ функцій $x_{s}: S \rightarrow \mathbb{R}$. Покладемо

$$
\begin{aligned}
& x_{0}(s)= \begin{cases}1, & s=0, \\
0, & s \in S \backslash\{0\},\end{cases} \\
& x_{n}(s)= \begin{cases}1-\frac{1}{n}, & s=0, \\
1+\frac{1}{n}, & s=n, \\
0, & s \in S \backslash\{0, n\},\end{cases}
\end{aligned}
$$


для кожного $n \in \mathbb{N}$ i

$$
x_{(n, m)}(s)= \begin{cases}1-\frac{1}{n}, & s=0, \\ \frac{1}{n}-\frac{1}{m}, & s=n, \\ \frac{1}{m}, & s=(n, m), \\ 0, & s \in S \backslash\{0, n,(n, m)\},\end{cases}
$$

для довільних $n, m \in \mathbb{N}$.

Тепер розглянемо простір $X=\left\{x_{s}: s \in S\right\}$ з частковою метрикою

$$
p(x, y)=\sum_{s \in S} \max \{x(s), y(s)\},
$$

тобто $(X, p)$ є підпростором $l_{1}(S)$.

Зауважимо, що $p\left(x_{0}, x_{0}\right)=p\left(x_{n, m}, x_{n, m}\right)=1$ для довільних $n, m \in \mathbb{N}$. Оскільки $p\left(x_{0}, x_{n}\right) \geq 2$ для кожного $n \in \mathbb{N}$, то

$$
U_{0}=B_{p}\left(x_{0}, \frac{1}{2}\right) \subseteq\left\{x_{0}\right\} \cup\left\{x_{n, m}: n, m \in \mathbb{N}\right\} .
$$

Тому $p(x, x)=1$ для кожного $x \in U_{0}$. Отже, звуження часткової метрики $p$ на діагональ $\Delta=\{(x, x): x \in X\}$ є неперервним в точці $\left(x_{0}, x_{0}\right)$ і згідно з твердженням 3.2 звуження $\pi_{p}$ неперервне в точці $x_{0}$.

Тепер покажемо, що $\overline{B_{p}\left(x_{0}, \varepsilon\right)} \nsubseteq U_{0}$ для довільного $\varepsilon>0$.

Зафіксуємо $\varepsilon>0$ і виберемо $n \in \mathbb{N}$ так, що $\frac{1}{n}<\varepsilon$. Тоді для довільного $m \geq n$ маємо

$$
p\left(x_{n, m}, x_{0}\right)=1+\frac{1}{m}+\frac{1}{n}-\frac{1}{m}=1+\frac{1}{n}<p\left(x_{0}, x_{0}\right)+\varepsilon .
$$

Тому $x_{n, m} \in B_{p}\left(x_{0}, \varepsilon\right)$.

Нехай $U=B_{p}\left(x_{n}, \delta\right)$, де $\delta>0$, - довільний базисний окіл точки $x_{n}$. Зауважимо, що

$$
p\left(x_{n, m}, x_{n}\right)=1-\frac{1}{n}+1+\frac{1}{n}-\frac{1}{m}=2+\frac{1}{m}=p\left(x_{n}, x_{n}\right)+\frac{1}{m} .
$$

Тому $x_{n, m} \in U$ при $m \geq \frac{1}{\delta}$.

Отже, $U \cap B_{p}\left(x_{0}, \varepsilon\right) \neq \emptyset$ і $x_{n} \in \overline{B_{p}\left(x_{0}, \varepsilon\right)} \backslash U_{0}$.

Теорема 4.2. Існуе метризовний частково метричний простір $(X, p)$ такий, що асоційоване відображення $\pi_{p}$ е розривним у кожній точиі $x \in X$.

Доведення. Для кожного $n \in \mathbb{N}$ через $S_{n}$ ми позначимо множину всіх наборів $\left(k_{1}, k_{2}, \ldots, k_{n}\right) \in \mathbb{N}^{n}$ таких, що $k_{1} \leq k_{2} \leq \cdots \leq k_{n}$. Крім того, для довільних $n \in \mathbb{N}, m \in \mathbb{N} 3 m \geq n$ i $\left(k_{1}, k_{2}, \ldots, k_{m}\right) \in S_{m}$ покладемо $\left.s\right|_{n}=\left(k_{1}, k_{2}, \ldots, k_{n}\right)$. Зрозуміло, що при цьому $\left.s\right|_{n} \in S_{n}$.

Покладемо $\alpha_{0}=1$. Індукцією відносно $n$ легко побудувати послідовність сімей $\left(\alpha_{s}: s \in S_{n}\right)$ чисел $\alpha_{s} \in(0,1)$, яка задовольняє такі умови: 
(a) послідовність $\left(\alpha_{k}\right)_{k=1}^{\infty}$ строго зростає і $\lim _{k \rightarrow \infty} \alpha_{k}<1$;

(b) для всіх $n \in \mathbb{N}$ i $\left(k_{1}, \ldots, k_{n}\right) \in S_{n}$ послідовність $\left(\alpha_{\left(k_{1}, \ldots, k_{n}, k_{n}+k\right)}\right)_{k=0}^{\infty}$ строго зростає i $\lim _{k \rightarrow \infty} \alpha_{\left(k_{1}, \ldots, k_{n}, k_{n}+k\right)}<\alpha_{\left(k_{1}, \ldots, k_{n}\right)}$;

(c) $\alpha_{\left(k_{1}, \ldots, k_{n}, k_{n}\right)}>\alpha_{\left(k_{1}, \ldots, k_{n}-1\right)}$ для всіх $n \in \mathbb{N}$ i $\left(k_{1}, \ldots, k_{n}\right) \in S_{n}$ таких, що $\left(k_{1}, \ldots, k_{n}-1\right) \in S_{n}$.

Позначимо $S_{0}=\{0\}, S=\bigcup_{n=0}^{\infty} S_{n}$. і побудуємо сім'ю $\left(x_{s}: s \in S\right)$ функцій $x_{s}: S \rightarrow \mathbb{R}$. Покладемо

$$
\begin{aligned}
x_{0}(s) & = \begin{cases}1, & s=0, \\
0, & s \in S \backslash\{0\},\end{cases} \\
x_{\left(k_{1}, \ldots, k_{n}\right)}(s) & = \begin{cases}\alpha_{\left(k_{1}, \ldots, k_{n}\right)}-\frac{1}{k_{1}}, & s=0, \\
\frac{1}{k_{1}}-\frac{1}{k_{2}}, & s=k_{1}, \\
\frac{1}{k_{2}}-\frac{1}{k_{3}}, & s=\left(k_{1}, k_{2}\right), \\
\ldots & s=\left(k_{1}, k_{2}, \ldots, k_{n-1}\right), \\
\frac{1}{k_{n-1}}-\frac{1}{k_{n}}, & s=\left(k_{1}, k_{2}, \ldots, k_{n}\right), \\
\frac{1}{k_{n}}, & s \in S \backslash\left\{0, k_{1},\left(k_{1}, k_{2}\right), \ldots,\left(k_{1}, \ldots, k_{n}\right)\right\}, \\
0, & \end{cases}
\end{aligned}
$$

для довільних $n \in \mathbb{N}$ i $\left(k_{1}, \ldots, k_{n}\right) \in S_{n}$.

Тепер, як і в доведенні попереднього твердження, розглянемо простір $X=\left\{x_{s}: s \in S\right\}$ з частковою метрикою

$$
p(x, y)=\sum_{s \in S} \max \{x(s), y(s)\} .
$$

Зауважимо, що $p\left(x_{0}, x_{0}\right)=1$ і $p\left(x_{s}, x_{s}\right)=\alpha_{s}$ для довільних $n \in \mathbb{N}$ i $s \in S_{n}$.

Зафіксуємо $n \in\{0,1,2, \ldots\}$ і $s=\left(k_{1}, k_{2}, \ldots, k_{n}\right) \in S_{n}$. Для кожного $m \in \mathbb{N}$ покладемо $t_{m}=\left(k_{1}, k_{2}, \ldots, k_{n}, k_{n}+m\right)$.

Легко бачити, що $x_{t_{m}} \rightarrow x_{s}$ в $X$. Але згідно з умовами (a) та (b) маємо

$$
\lim _{m \rightarrow \infty} p\left(x_{t_{m}}, x_{t_{m}}\right)=\lim _{m \rightarrow \infty} \alpha_{t_{m}}<\alpha_{s}=p(x, x) .
$$

Тому згідно з твердженням 3.2 відображення $\pi_{p}$ не $€$ неперервним в точці $x$.

Залишилось перевірити, що $X$ - метризовний. Оскільки простір $X$ зліченний з першою аксіомою зліченності, то згідно з [4, Теорема 4.2.9] достатньо показати, що $X$ регулярний. 
Спочатку перевіримо регулярність простору $X$ в точці $x_{0}$. Зауважимо, що

$$
p\left(x_{0}, x_{s}\right)=1+\frac{1}{k_{1}}=p\left(x_{0}, x_{0}\right)+\frac{1}{k_{1}}
$$

для довільних $n \in \mathbb{N}$ i $s=\left(k_{1}, k_{2}, \ldots, k_{n}\right) \in S_{n}$. Зафіксуємо $m \in \mathbb{N}$ i розглянемо окіл

$$
U=B_{p}\left(x_{0}, \frac{1}{m}\right)=\left\{x_{0}\right\} \cup\left\{x_{\left(k_{1}, \ldots, k_{n}\right)}: k_{1}>m\right\}
$$

точки $x_{0}$.

Нехай $x=x_{\left(k_{1}, \ldots, k_{n}\right)} \in X \backslash U$. Тоді $k_{1} \leq m$ і $\alpha_{\left(k_{1}, \ldots, k_{n}\right)} \leq \alpha_{k_{1}} \leq \alpha_{m}$ згідно з умовою (b). Розглянемо окіл $V=B_{p}\left(x, \frac{1}{m}-\frac{1}{m+1}\right)$ точки $x$. Для довільного $y \in V$ маємо

$$
y(0)<x(0)+\frac{1}{m}-\frac{1}{m+1}=\alpha_{\left(k_{1}, \ldots, k_{n}\right)}-\frac{1}{k_{1}}+\frac{1}{m}-\frac{1}{m+1} \leq \alpha_{m}-\frac{1}{m+1} .
$$

3 іншого боку, для довільного $z=x_{\left(l_{1}, \ldots, l_{i}\right)} \in U$ маємо $l_{1} \geq m+1 \mathrm{i}$ $\alpha_{\left(l_{1}, \ldots, l_{i}\right)}>\alpha_{m}$ згідно з (c). Тому

$$
z(0)=\alpha_{\left(l_{1}, \ldots, l_{i}\right)}-\frac{1}{l_{1}}>\alpha_{m}-\frac{1}{m+1} .
$$

Отже, $V \cap U=\emptyset$ і множина $U$ - відкрито-замкнена. Таким чином, простір $X$ регулярний в точці $x_{0}$. Крім того, для кожного $k \in \mathbb{N}$ множина

$$
G_{k}=\left\{x \in X: x=x_{\left(k, k_{2}, \ldots, k_{n}\right)}\right\}=\left\{x_{s} \in X \backslash\left\{x_{0}\right\}:\left.s\right|_{1}=k\right\}
$$

відкрито-замкнена, як різниця відкрито-замкнених множин.

Покажемо регулярність простору $X$ в кожній точці $x \in\left\{x_{s}: s \in S_{1}\right\}$. Зафіксуємо $k \in S_{1}$. Зауважимо, що

$$
p\left(x_{k}, x_{s}\right)=\alpha_{k}+\frac{1}{k_{2}}=p\left(x_{k}, x_{k}\right)+\frac{1}{k_{2}}
$$

для кожного $x_{s} \in G_{k} \backslash\left\{x_{k}\right\}$, де $s=\left(k, k_{2}, \ldots, k_{n}\right) \in S_{n}$ i $n \geq 2$. Зафіксуємо $m \geq k$ і розглянемо окіл

$$
U=G_{k} \cap B_{p}\left(x_{k}, \frac{1}{m}\right)=\left\{x_{k}\right\} \cup\left\{x_{\left(k, k_{2}, \ldots, k_{n}\right)}: k_{2}>m\right\}
$$

точки $x_{k}$.

Нехай $x=x_{\left(k, k_{2}, \ldots, k_{n}\right)} \in G_{k} \backslash U$. Тоді $k \leq k_{2} \leq m$ i

$$
\alpha_{\left(k, k_{2}, \ldots, k_{n}\right)} \leq \alpha_{\left(k, k_{2}\right)} \leq \alpha_{(k, m)}
$$

згідно з умовою (b). Розглянемо окіл $V=G_{k} \cap B_{p}\left(x, \frac{1}{m}-\frac{1}{m+1}\right)$ точки $x$. Для довільного $y \in V$ маємо

$$
\begin{aligned}
y(0)+y(k) & <x(0)+x(k)+\frac{1}{m}-\frac{1}{m+1} \\
& =\alpha_{\left(k, k_{2}, \ldots, k_{n}\right)}-\frac{1}{k_{2}}+\frac{1}{m}-\frac{1}{m+1} \\
& \leq \alpha_{(k, m)}-\frac{1}{m}+\frac{1}{m}-\frac{1}{m+1} \\
& =\alpha_{(k, m)}-\frac{1}{m+1} .
\end{aligned}
$$


3 іншого боку, для довільного $z=x_{\left(k, l_{2}, \ldots, l_{i}\right)} \in U$ маємо $l_{2} \geq m+1$ i $\alpha_{\left(k, l_{2}, \ldots, l_{i}\right)}>\alpha_{(k, m)}$ згідно з (c). Тому

$$
z(0)+z(k)=\alpha_{\left(k, l_{2}, \ldots, l_{i}\right)}-\frac{1}{l_{2}}>\alpha_{(k, m)}-\frac{1}{m+1} .
$$

Отже, $V \cap U=\emptyset$ і множина $U$ - відкрито-замкнена. Таким чином, простір $X$ регулярний в точці $x_{k}$. Зауважимо, що, крім того, для кожного $s \in S_{2}$ множина

$$
G_{s}=\left\{x_{t} \in X:\left.t\right|_{2}=s\right\}
$$

відкрито-замкнена, як різниця відкрито-замкнених множин. Тепер для кожного $n \geq 3$ і $s \in S_{n}$ покладемо

$$
G_{s}=\left\{x_{t} \in X:\left.t\right|_{n}=s\right\} .
$$

Далі, використовуючи індукцію відносно $n$ нескладно можна довести регулярність простору $X$ в кожній точці $x \in\left\{x_{s}: s \in S_{n}\right\}$ і відкритозамкненість кожної множини $G_{s}$, де $s \in S_{n}$. При цьому індуктивний перехід доводиться повністю аналогічно, як у випадку $n=2$.

\section{5. ВІДОБРАЖЕННЯ ЗІ ЗНАЧЕННЯМИ У ЧАСТКОВО МЕТРИЧНИХ ПРОСТОРАХ}

Для топологічних просторів $X$ і $Y$ через $C(f)$ ми позначатимемо множину точок неперервності відображення $f: X \rightarrow Y$, а через $D(f)$ ми позначатимемо множину точок розриву відображення $f$. Добре відомо, що множина точок неперервності відображення зі значеннями у метризовному просторі має тип $G_{\delta}$, а множина точок розриву має тип $F_{\sigma}$. Наступний результат є певним аналогом цього факту для відображень зі значеннями у частково метричному просторі.

Теорема 5.1. Нехай $X$ - топологічний простір, $(Y, p)$ - частково метричний простір $i f: X \rightarrow Y$ такі, що для довільної ніде не щільноӥ множини $B \subseteq Y$ множина $f^{-1}(B)$ ніде не щільна в $X$. Тод $i$ існують $G_{\delta}$-множина $C$ в $X$ і множина $A$ першої категоріӥ в $X$ такі, що $C(f)=C \cup A$.

Доведення. Зафіксуємо $n \in \mathbb{N}$ і позначимо через $B_{n}$ множину всіх точок $y \in Y$ таких, що $p(y, y) \leq n$ і коливання звуження часткової метрики $p$ на діагональ $\Delta=\{(y, y): y \in Y\}$ у точці $(y, y)$ не менше, ніж $\frac{1}{n}$. Застосуємо твердження 3.4 до функції $\varphi_{n}: Y \rightarrow[-(n+1), n+1]$,

$$
\varphi_{n}(y)= \begin{cases}-(n+1), & p(y, y)<-(n+1), \\ n+1, & p(y, y)>n+1, \\ p(y, y), & |p(y, y)| \leq n+1,\end{cases}
$$


і одержимо, що множина $B_{n}$ ніде не щільна в $Y$. Тому множина

$$
A_{n}=f^{-1}\left(\overline{B_{n}}\right)
$$

також ніде не щільна в $X$. Далі покладемо $G_{n}=X \backslash \overline{A_{n}}$ і розглянемо відображення $f_{n}: G_{n} \rightarrow\left(Y, d_{p}\right)$. Позначимо через $C_{n}$ множину всіх точок $x \in G_{n}$ таких, що $\omega_{f_{n}}(x)<\frac{1}{n}$.

Тепер покладемо $C=\bigcap_{n=1}^{\infty} C_{n}$ і $A=C(f) \bigcap\left(\bigcup_{n=1}^{\infty} \overline{A_{n}}\right)$. Оскільки всі множини $G_{n}$ відкриті, то відображення $g: X \rightarrow\left(Y, d_{p}\right), g(x)=f(x)$, неперервне в кожній точці множини $C$. Тому і відображення $f$ неперервне в кожній точці множини $C$. Залишилось показати, що $f$ розривне в кожній точці

$$
x_{0} \in\left(X \backslash \bigcup_{n=1}^{\infty} \overline{A_{n}}\right) \backslash C=\left(\bigcap_{n=1}^{\infty} G_{n}\right) \backslash C .
$$

Зауважимо, що $g$ розривне в точці $x_{0}$ і $y_{0}=f\left(x_{0}\right) \in Y \backslash\left(\bigcup_{n=1}^{\infty} B_{n}\right)$. Тому звуження часткової метрики на $\Delta \epsilon$ неперервним в точці $\left(y_{0}, y_{0}\right)$ і згідно з твердженням 3.2 асоційоване з $p$ відображення $\pi_{p}$ неперервне в точці $y_{0}$. Отже, відображення $f$ розривне в точці $x_{0}$.

Наступний приклад вказує на істотність додаткових умов на відображення $f$ у теоремі 5.1.

Твердження 5.2. Існуе регулярний частково метричний простір $(Y, p)$ $i$ відображення $f:[0,1] \rightarrow Y$ такі, що множина $C(f)$ невимірна за Борелем.

Доведення. Нехай $A \subseteq[0,1]$ - невимірна за Лебегом множина така, що множини $A$ і $B=[0,1] \backslash A$ щільні в $[0,1]$. Для кожного $a \in A$ розглянемо функцію $y_{a}:[0,2] \rightarrow \mathbb{R}$,

$$
y_{a}(t)= \begin{cases}2, & t \in[a, a+1] \\ 0, & t \in[0,2] \backslash[a, a+1]\end{cases}
$$

а для кожного $b \in B$ розглянемо функцію $y_{b}:[0,2] \rightarrow \mathbb{R}$,

$$
y_{b}(t)= \begin{cases}1, & t \in[b, b+1] \\ 0, & t \in[0,2] \backslash[b, b+1]\end{cases}
$$


Покладемо $Y=\left\{y_{x}: x \in[0,1]\right\}$ і розглянемо простір $Y$ з частковою метрикою

$$
p(y, z)=\int_{0}^{2} \max \{y(t), z(t)\} d \mu(t) .
$$

Зауважимо, що

$$
p\left(y_{u}, y_{v}\right)-p\left(y_{u}, y_{u}\right) \geq|u-v|
$$

для довільних $u, v \in[0,1]$. Тому простір $(Y, p)$ регулярний.

Розглянемо відображення $f:[0,1] \rightarrow Y, f(x)=y_{x}$. Безпосередньою перевіркою легко переконатися, що $C(f)=A$.

Відображення $f: X \rightarrow Y$ між топологічними просторами $X$ і $Y$ називається $F_{\sigma}$-вимірною, якщо для кожної відкритої в $X$ множини $G$ множина $f^{-1}(G)$ має тип $F_{\sigma}$ в $X$.

Наступний результат узагальнює теорему про $F_{\sigma}$-вимірність напівнеперервної функції (дивись [8, Глава XV, § 3-4]).

Теорема 5.3. Припустимо, що $X$ - досконало нормальний простір, $(Y, p)$ - частково метричний простір, такий що $\left(Y, d_{p}\right)$ сепарабельний, i $f: X \rightarrow(Y, p)$ - неперервне відображення. Тоді відображення $g: X \rightarrow\left(Y, d_{p}\right), g(x)=f(x)$, е $F_{\sigma}$-вимірним.

Доведення. Для довільних $y_{0} \in Y$ i $\varepsilon>0$ покладемо

$$
G\left(y_{0}, \varepsilon\right)=B_{p}\left(y_{0}, \varepsilon\right) \backslash\left\{y \in Y: p(y, y)<p\left(y_{0}, y_{0}\right)+\varepsilon\right\} .
$$

Зауважимо, що

$$
B_{d_{p}}\left(y_{0}, \varepsilon\right) \subseteq G\left(y_{0}, \varepsilon\right) \subseteq B_{d_{p}}\left(y_{0}, 3 \varepsilon\right) .
$$

Тому довільну відкриту множину $G$ в просторі $\left(Y, d_{p}\right)$ можна подати у вигляді об'єднання множин $G(y, \varepsilon)$. Причому, оскільки простір $\left(Y, d_{p}\right)$ сепарабельний, то множину $G$ можна подати у вигляді

$$
G=\bigcup_{n=1}^{\infty} G\left(y_{n}, \varepsilon_{n}\right) .
$$

Залишилось зауважити, що кожна множина $f^{-1}\left(G\left(y_{n}, \varepsilon_{n}\right)\right)$ має тип $F_{\sigma}$, як прообраз при неперервному відображенні різниці двох відкритих множин.

\section{ЛiтерАтурА}

[1] I. Altun, H. P. Masiha, F. Sabetghadam. Fixed point theorems for integral type contractions on partial metric spaces. Ukr. Mat. Zhurn., 68(6):826-834, 2016.

[2] I. Altun, F. Sola, H. Simsek. Generalized contractions on partial metric spaces. Topology and its Applications, 157(18):2778-2785, 2010. 
[3] L. Ciric, B. Samet, H. Aydi, Vetro C. Common fixed points of generalized contractions on partial metricc spaces and an application. Appl. Math. Comput., 218:2398-2406, 2011.

[4] R. Engelking. General Topology. Heldermann Verlag, Berlin, 1989.

[5] E. Karapinar, I. M. Erhan. Fixed point theorems for operators on partial metricc spaces. Appl. Math. Lett., 24:1894-1899, 2011.

[6] S. G. Matthews. Partial metric space. 8th British Colloquium for Theoretical Computer Science, March 1992. In Research Report 212, Dept. of Computer Science, University of Warwick, 1992.

[7] S. G. Matthews. Partial metric topology. Proc. 8th Summer Conference on General Topology and Applications, Ann. New York Acad. Sci., 728:183-197, 1994.

[8] I. P. Natanson. Theory of Functions of a real variable. Dover Books on Mathematics. Dover Publications, 2016.

[9] S. Romaguera. A kirk type characterization of completeness for partial metric spaces. Fixed Points Theory Appl., page 10 p., 2010.

[10] J. E. Stoy. Denotational semantics: the Scott-Strachey approach to programming language theory. MIT Press. Cambridge Massachusetts, 1977.

Надійшла до редакиії 16 листопада 2016, прийнята до друку 12 січня $201 \%$.

Мироник Вадим Ілліч

58012, КАФЕДРА АЛГЕБРИ ТА ІНФОРМАТИКИ, ЧЕРНІВЕЦЬКИЙ НАЦІОНАЛЬНИЙ УНІВЕРСИТЕТ, ВУЛ. КОЦЮБИНСЬКОГО 2, М. ЧЕРНІвцІ, УКРАЇНА

Email: vadmyron@gmail.com

Михайлюк Володимир Васильович

58012, КАФЕДРА МАТЕМАТИЧНОГО АНАЛІЗУ, ЧЕРНІВЕЦЬКИЙ НАЦІОНАЛЬНИЙ УНІВЕРСИТЕТ, ВУЛ. КОЦЮБИНСЬКОГО 2, М. ЧЕРнІвцІ, УКРАЇнА

Email: vmykhaylyuk@ukr.net 